\title{
Core Level Photelectron and X-Ray Absorption Spectroscopy of Free Argon Clusters: Size-Dependent Energy Shifts and Determination of Surface Atom Coordination
}

\author{
O. Björneholm, F. Federmann, F. Fössing, and T. Möller \\ Hamburger Synchrtronstrahlungslabor (HASYLAB) am Deutschen Elektronen Synchrotron (DESY), \\ Notkestrasse 85, D-22603 Hamburg, Germany
}

(Received 15 April 1994)

\begin{abstract}
Core level photoelectron and $\mathrm{x}$-ray absorption spectra for free argon clusters from $\langle N\rangle \approx 5$ to $\langle N\rangle \approx 4000$ are presented. Spectral features originating from surface and bulk sites of the clusters are identified. These are seen to shift with cluster size. From the development of the spectra from the isolated atom to the largest clusters, information about both the size-dependent cluster-specific electronic structure and that of the "infinite" solid is obtained. Using a simple model for the core level binding energy shifts, effective surface coordination numbers are derived. These range from 5.3 for $\langle N\rangle \approx 10$ to 8.5 for the solid.
\end{abstract}

PACS numbers: 73.61.Ng, 36.40.-c, 78.70.Dm, 79.60.Bm

Clusters can be seen as polyatomic aggregates between isolated atoms and bulk solids, in which a considerable part of the atoms are located in surface sites of low coordination numbers [1-3]. The nature and distribution of these atomic sites vary with cluster size, and many of the most interesting properties of clusters are intimately connected to this interplay between the size-dependent geometry and the electronic structure. This also makes clusters interesting for applications where the tuning of various physical and chemical properties could be achieved by cluster size selection. In the cases of atoms, molecules, surfaces, and solids, the connection between the electronic and the geometric structure has been extensively studied using core level spectroscopic techniques such as $\mathrm{X}$-ray photoelectron (XP) and x-ray absorption (XA) spectroscopy $[4,5]$. Unlike valence levels, core levels, even in solids, are atomiclike and highly localized, and core level spectra therefore reflect local properties. Consequently, spectral features corresponding to atoms with different local environment, such as atoms in bulk and different surface sites of solids, can often be well separated [6]. Core level spectroscopy is therefore highly interesting to apply to the study of free clusters. This has, however, been difficult due to intensity problems, and only recent XA studies have shown it to be feasible $[7,8]$.

In this Letter we present XP spectra of the $2 p$ core level of free argon clusters. To the best of our knowledge these are the first core level XP spectra reported for free clusters. These data are combined with high-resolution, cluster-specific XA spectra. With both spectroscopies clearly separated, bulk and surface features are identified. From the development of the spectra with cluster size, information not only about cluster specific properties but also about the solid is obtained. Using a simple model for the binding energy shifts, information about the effective surface coordination numbers is derived.

Argon clusters were produced in an adiabatic expansion of argon gas through a conical nozzle (diameter $0.1 \mathrm{~mm}$, opening angle $2 \theta=30^{\circ}$ ). The beam consisted of a mixture of atoms and clusters, characterized by an average cluster size of $\langle N\rangle$ atoms per cluster and a width of approximately $N$. The average cluster size was changed by variations of the stagnation pressure and nozzle temperature, and was derived using scaling laws [9-11]. The uncertainty in the absolute size determination is of the order of a factor of 2, depending on which of the published scaling laws are used. We have, in this case, followed Ref. [11]. The XA and XP spectroscopy measurements were performed at the high-flux HASYLAB BW3 undulator beam line [12]. The spectra were recorded with time-of-flight (TOF) techniques, using the arrival of the photoion or photoelectron and the following synchrotron radiation pulse as start and stop signals, respectively. For the photoions, a very short TOF mass spectrometer of $10 \mathrm{~mm}$ total flight length was used [13]. The "cluster" beam contains a significant amount of uncondensed atoms, especially at low $\langle N\rangle$ conditions, but by monitoring the partial yield of cluster ions [14], cluster-specific argon XA spectra in the $2 p$ region with a resolution of approximately $0.05 \mathrm{eV}$ could be recorded without atomic contributions. In comparison to the simultaneously recorded $\mathrm{Ar}^{2+}$ yield, which is almost exclusively of atomic origin, the relative photon energies could be calibrated to within $\pm 0.01 \mathrm{eV}$. A second and longer $(150 \mathrm{~mm})$ TOF spectrometer was used for photoelectron detection. Argon $2 p$ photoelectron spectra from both atoms and clusters were simultaneously recorded with a total resolution better than $0.3 \mathrm{eV}$.

Figure 1 shows a series of argon $2 p$ XA spectra for various average cluster sizes $\langle N\rangle$, including the atom and the solid. The spectrum of the atom is due to transitions from the $2 p$ core level to unoccupied Rydberg orbitals. The lowest-energy transition $2 p_{3 / 2} \rightarrow 4 s$ is located at $244.39 \mathrm{eV}$ [15]. This is followed by the spin-orbit partner $2 p_{1 / 2} \rightarrow 4 s$ at $2.2 \mathrm{eV}$ higher energy, above which $2 p_{3 / 2} \rightarrow n d$ transitions dominate. In the clusters, the lower 


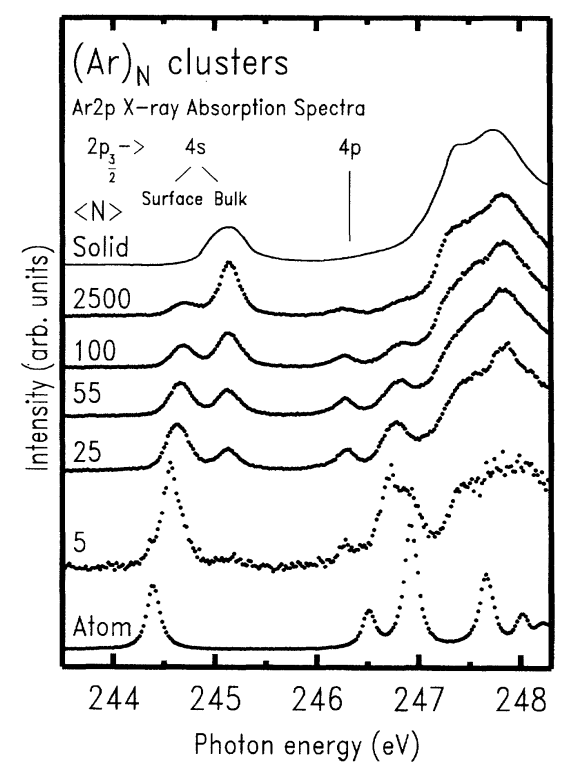

FIG. 1. A selection of argon $2 p$ XA spectra for argon clusters of various average sizes $\langle N\rangle$. The spectrum of the solid is taken from Ref. [16].

excitations are still observed as lines, whereas the higher ones form broader features. The clearest changes are observed for the $2 p_{3 / 2} \rightarrow 4 s$ transition, which is split into two separate components at somewhat higher energy. The low-energy component dominates at small cluster sizes, whereas the higher one gradually becomes more prominent with increasing cluster size. The high-energy line is the only one observed in the spectrum of the argon solid, in which it has been attributed to a transition into a bulk core exciton state associated to the atomic $2 p_{3 / 2}^{-1} 4 s$ Rydberg state $[16,17]$. The lower of the lines is observed to shift upward in energy from $244.58 \mathrm{eV}(+0.19 \mathrm{eV}$, values in brackets give the shift relative to the atomic $2 p_{3 / 2} \rightarrow$ $4 s$ transition) for the smallest cluster size to $244.70 \mathrm{eV}$ $(+0.31 \mathrm{eV})$ for the largest. No comparable shift is observed for the high-energy line, which has an energy of $245.14 \mathrm{eV}(+0.75 \mathrm{eV})$ varying less than $\pm 0.01 \mathrm{eV}$ over the whole size range. The $2 p_{3 / 2} \rightarrow 4 s$ transition energy as a function of cluster size is shown in Fig. 2.

Some geometric considerations are helpful for the interpretation of the spectra. As the cluster size increases, the fraction of atoms located at the cluster surface will gradually decrease, whereas the bulk fraction will increase. The coordination of the individual argon atoms will also change, from 0 for the isolated atom to 8 or 9 for an atom in the surface of solid argon and 12 for an atom located in the bulk. In the atom, the final $2 p_{3 / 2}^{-1} 4 s$ Rydberg state has an appreciably larger spatial extent than the ground state, but unlike in more strongly bonded systems such as semiconductors and metals, hybridization with orbitals on the neighboring atoms is practically negligible in this case. The effect of the coordination

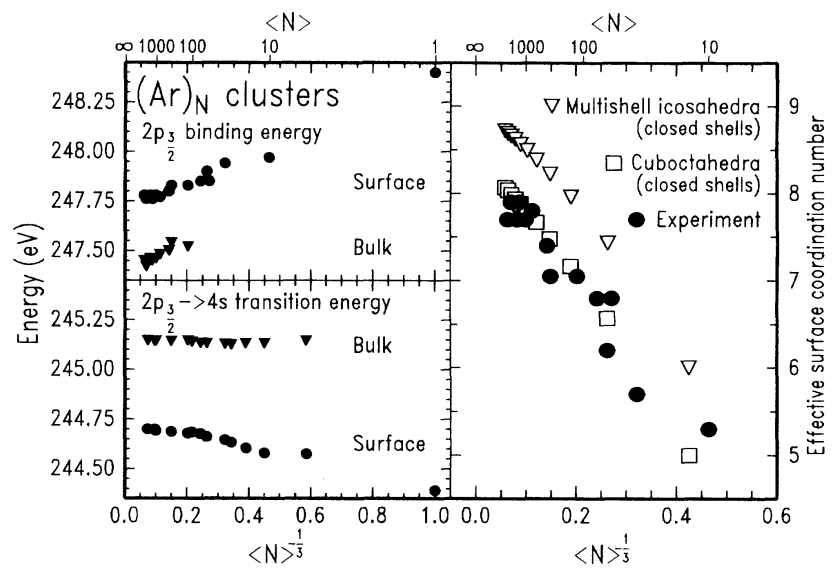

FIG. 2. Experimental surface and bulk $2 p_{3 / 2} \rightarrow 4 s$ transition energies (lower left) and $2 p_{3 / 2}$ binding energies (upper left) as a function of $\langle N\rangle^{-1 / 3}$, respectively, together with effective surface coordination numbers (right).

has been described as a "spatial restriction" on the final state valence orbital, resulting in an increase in energy $[14,16,18,19]$. In a simplified picture the excited state orbital is compressed by the surrounding atoms, leading to an increase in the valence orbital energy in a way analogous to the case of a particle confined in a shrinking box. From this analogy it follows that the excitation energy increases with increasing coordination [20]. Based on the intensity dependence on cluster size and on the energy of the transitions we therefore interpret the lowenergy line as arising from atoms located at the cluster surface, and the high-energy line as corresponding to bulk atoms. As seen in Fig. 2, the bulk component has a practically constant energy over the whole cluster size range. As soon as the first coordination shell is closed, the bulk component can be observed at the same energy as for the "infinite" solid. From this it follows that only the first coordination shell has any appreciable influence on the $2 p_{3 / 2} \rightarrow 4 s$ transition. The upward shift of the surface component can thus be understood as due to a gradual increase in the coordination number of the surface sites. In this sense there is a gradual transition from the Rydberg state of the atom to the exciton state of the solid.

The development of the XA spectra thus facilitates the understanding of the atomic origins of the solid state spectral features. For the clusters, the reduced but size-dependent coordination in surface sites is manifested in energy shifts. When fully understood, these effects may yield further information about the local geometry in these sites. Already, at the present qualitative level of understanding, however, the size-dependent shift of the $2 p_{3 / 2} \rightarrow 4 s$ surface component can be connected to a gradual increase in the coordination number for the surface sites with cluster size. We will now turn to the core level XP spectra, from which more quantitative information about the surface coordination can be derived. 
Figure 3 shows a selection of XP spectra of the argon $2 p$ region for various average cluster sizes $\langle N\rangle$. Note that, in contrast to the above XA spectra, the XP spectra are not cluster specific, but contain contributions from both free atoms and clusters. In the spectrum of the atom the $2 p_{1 / 2}$ and $2 p_{3 / 2}$ spin-orbit split lines are observed at 248.4 and $250.6 \mathrm{eV}$ binding energy, respectively [21]. The asymmetric line shape is due to the instrument function of the TOF photoelectron spectrometer in combination with post-collision-interaction effects. At small cluster sizes, additional features are observed on the low binding energy side of the atomic lines. As the cluster size increases, the nonatomic lines are seen to gradually shift towards lower binding energy, and split into two separate lines. The lower of these two lines becomes gradually more prominent as the cluster size increases. For the largest size, the nonatomic components have 0.62 and $0.95 \mathrm{eV}$ lower binding energies than the atomic component, respectively.

In solid argon, the atoms surrounding the core ionized site will be polarized. Because of this polarization screening of the ionic final state, the argon $2 p$ binding energy will be lower in the clusters than in the isolated atom. The spectral features on the low binding energy side of the atomic peaks can thus be attributed to clusters. The efficiency of the polarization screening decreases rapidly with distance, and the nearest neighbors are therefore the most important ones for the screening. Similar to the above XA spectra, the two separate lines at large cluster sizes can therefore be attributed to atoms located in the bulk and at the surface of the clusters, respectively. The spectra were thus decomposed into atomic, surface, and bulk components,

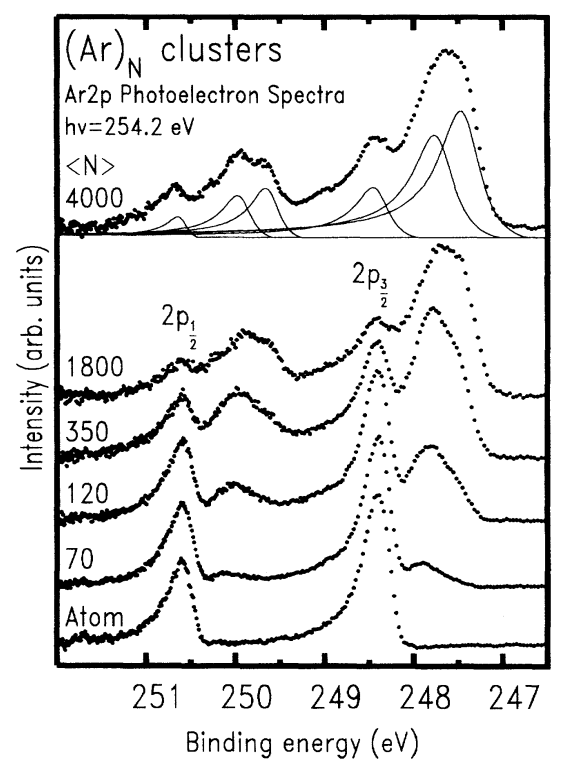

FIG. 3. A selection of argon $2 p$ XP spectra for argon clusters of various average sizes $\langle N\rangle$. The decomposition into atomic, surface, and bulk components is indicated in the top spectrum. as indicated in the top spectrum of Fig. 3. The bulk and surface $2 p_{3 / 2}$ binding energies are shown in Fig. 2 as function of cluster size. Since a distribution of cluster sizes is present in the beam, and since different surface sites and "bulk" atoms located at different distances from the center contribute to the spectral features for each mass, these values should be regarded as the position of the respective envelopes of the site and size distribution. In a classical polarization screening model the cluster size dependent term is proportional to $N^{-1 / 3}[2,22]$. By extrapolating the binding energies to $\langle N\rangle^{-1 / 3}=0$, the cluster data can thus be used to derive information about the solid. This yields a $2 p_{3 / 2}$ binding energy of $247.4 \pm 0.05 \mathrm{eV}$ for bulk atoms in solid argon, i.e., a shift of $-1.0 \pm 0.05 \mathrm{eV}$ compared to the free atom. Previously published values of this shift are widely scattered: $-2.4 \mathrm{eV}$ [16], -1.0 to $-1.4 \mathrm{eV}$ [19], $-1.0 \mathrm{eV}$ [23], and $+0.1 \mathrm{eV}$ [17]. As the present measurements, in contrast to the previous ones, do not suffer from problems with, e.g., sample charging or nonresolved bulkplus-surface features, they are clearly preferable. For surface atoms the extrapolation procedure yields a binding energy of $247.7 \pm 0.05 \mathrm{eV}$. The binding energy difference between atoms located at the surface and in the bulk, commonly denoted the surface shift, is $0.3 \mathrm{eV}$ for solid argon. Since surface atoms have a lower coordination than bulk atoms, the surface shift suggests a connection between the binding energy shift and the local coordination. We will now make use of this to study the local coordination at the cluster surface.

At the cluster surface, atoms are located in various sites with different coordination numbers, e.g., in corners, edges, etc. The relative frequencies of these vary with cluster size and between different structural models [24]. By comparing the binding energies for atoms that are free, located at the cluster surface and in the bulk of the solid, information about the effective coordination number on the surface can be derived. This is done by scaling the twelvefold coordination of the bulk atoms with the ratio of the atom-to-cluster surface and atom-to-solid bulk binding energy shifts. This simple model can be justified in the following way: Even though the electric field of the ion extends beyond the nearest neighbors, the first coordination shell gives rise to $\approx 65 \%$ of the polarization screening. With two more shells, $85 \%$ of the maximal screening is reached. For an atom at the surface, the nearest neighbors can be thought of as defining a solid angle in which polarization screening takes place. Already for small clusters with a mean size around 50, there are for a surface atom on the order of two to three atomic layers beyond the nearest neighbors. For a certain cluster size, a surface atom on average has more coordination shells within the solid angle defined by the number of nearest neighbors than a bulk atom, justifying the use of the solid bulk binding energy in this simple model. The errors introduced by using a linear model of the shift as a function of the number of nearest neighbors should be in the order of or smaller 
than $15 \%$ for all the studied cluster sizes, except the smallest one [20]. The derived numbers are averages over the occupation frequency of the various surface sites, and we will denote these effective coordination numbers. In Fig. 2, these effective coordination numbers of atoms at the surface of the cluster are seen to vary from 5.3 in the smallest clusters $(\langle N\rangle \approx 10)$ to 7.8 in the largest clusters $(\langle N\rangle \approx 4000)$. Note that for $\langle N\rangle<100$, the bulk peak could not be separated in the spectra, and the effective coordination number does then refer to the whole cluster.

Several different structural models have been proposed for rare gas clusters [25]. Of particular interest are the multishell icosahedral structures with pentagonal symmetry, favored for small clusters, and the cuboctahedral truncation of the bulk fcc structure, favored for large clusters. The transition between the two structures has been placed somewhere between $N=750$ and $N=10000$ [25,26]. The average surface coordination numbers for these two models are included in Fig. 2. These are only well defined for the closed shell sizes $N=13,55,147, \ldots$, while for intermediate sizes the values depend on which surface sites happen to be occupied. The average surface coordination number will then be lower than for the closed shell sizes. The experimental values show the same general trend as the models, and converge towards a value of approximately 8.5 , in good agreement with the value of 8.4 expected for the cuboctahedral structure. Considering the relatively small differences in surface coordination numbers between the two structures, the simplicity of the binding energy shift model and the complication of open shell clusters we do, however, not think that any definite decision can be made between the two structures at this point. For other systems in which the shifts are more strongly dependent on the nearest neighbors and where there are more marked coordination number differences, this method should, however, give direct structural information. Some highly interesting examples of such systems are covalently bonded clusters such as $\mathrm{Si}_{n}, \mathrm{C}_{n}$, and "Metcars" [27].

In conclusion, we have shown how the development of the core level spectra from the isolated atom to large clusters can be used to derive information about both clusters and the infinite solid. One particular advantage of core level spectroscopy compared to valence level spectroscopy is its ability to probe local structure, e.g., the coordination of surface atoms. This was used to show how the transition from atomic Rydberg states to the exciton states of the solid is directly connected to the increase in local coordination. Quantitative information about such an increase in coordination for surface sites was derived from the core level photoelectron spectra. These results illustrate how, at the surface of clusters, the electronic structure changes with the geometric structure, thus pointing forward in the direction of the tuning of physical and chemical properties by cluster size selection.

A. Ding, P. Stampfli, and E. Rühl are acknowledged for stimulating discussions and making results available prior to publication. O. B. would like to acknowledge the EU for a HCM postdoctoral fellowship.

[1] M. R. Hoare, Adv. Chem. Phys. 40, 49 (1979).

[2] J. Jortner, Z. Phys. D 24, 247 (1992).

[3] J. Stapelfeldt et al., Phys. Rev. Lett. 62, 98 (1989).

[4] K. Siegbahn et al., ESCA Applied to Free Molecules (North-Holland, Amsterdam, 1969).

[5] J. Stöhr, NEXAFS Spectroscopy (Springer, Berlin, 1992).

[6] A. Flodström et al., in Synchrotron Radiation Research, edited by R.Z. Bachrach (Plenum Press, New York, 1992), and references therein.

[7] E. Rühl et al., Chem. Phys. Lett. 178, 558 (1991).

[8] F. Federmann et al., Phys. Rev. Lett. 73, 1549 (1994).

[9] O. F. Hagena et al., J. Chem. Phys. 56, 1793 (1972).

[10] O.F. Hagena, Z. Phys. D 4, 291 (1987).

[11] R. Karnbach et al., Rev. Sci. Instrum. 64, 2838 (1993).

[12] C. U.S. Larsson et al., Nucl. Instrum. Methods Phys. Res., Sect. A 337, 603 (1994)

[13] A. Beutler, Diplomarbeit, Hamburg, 1993.

[14] E. Rühl et al., J. Chem. Phys. 98, 2653 (1993). In the case of absorption of photons with energies well above the double ionization threshold, fragmentation produces predominantly $\mathrm{Ar}_{2}{ }^{+}$, the most stable ions. The yield of these were used to measure the absorption of clusters in the beam.

[15] G. C. King et al., J. Phys. B 10, 2479 (1977).

[16] R. Haensel et al., J. Phys. C 4, 236 (1971).

[17] M. Altarelli et al., Solid State Commun. 16, 143 (1975).

[18] O. Björneholm et al., Phys. Rev. B 47, 2308 (1993).

[19] W. Wurth et al., Phys. Rev. B 47, 6697 (1993).

[20] This qualitative argument is supported by recent calculations by P. Stampfli (private communication).

[21] G.P. Williams, in X-Ray Data Booklet, edited by J. Kirz and D. Vaughan (Lawrence Berkeley Laboratory, University of California, Berkeley, California, 1986).

[22] P. Stampfli et al., Ber. Bunsen-Ges. Phys. Chem. 96, 1243 (1992).

[23] A. Knop et al., Chem. Phys. Lett. 223, 553 (1994).

[24] R. E. Benfield, J. Chem. Soc. Faraday Trans. 88, 1107 (1992).

[25] J. Farges et al., Surf. Sci. 106, 95 (1981).

[26] B. W. van de Waal, J. Chem. Phys. 98, 4909 (1993).

[27] B. C. Guo et al., Science 254, 1144 (1992). 